\title{
Approximating Vector Quantization by Transformation and Scalar Quantization
}

\author{
Lei Yang, Pengwei Hao and Dapeng Wu
}

\begin{abstract}
Vector quantization provides better ratedistortion performance over scalar quantization even for a random vector with independent dimensions. However, the design and implementation complexity of vector quantizers is much higher than that of scalar quantizers. To reduce the complexity while achieving performance close to optimal vector quantization and better than scalar quantization, we propose a new quantization scheme, which consists of a transformation and scalar quantization. The transformation is to convert a two-axis representation to a triaxis representation; then scalar quantization is applied to each of the three axes. The proposed quantizer is asymptotically optimal/suboptimal for low/high rate quantization, especially for the quantization with certain prime number of quantization levels. The proposed quantizer has $O\left(N^{2}\right)$ design complexity, while VQ has $O(N !)$ design complexity, where $N$ is the number of quantization levels per dimension. The experimental results show that it achieves average bit-rate saving of $0.4 \%-24.5 \%$ over restricted/unrestricted polar quantizers and rectangular quantizers for signals of circular and elliptical Gaussian distributions and Laplace distributions. It holds potential of improving the performance of existing image and video coding schemes.
\end{abstract}

Index Terms-Scalar quantization, Vector quantization, Hexagonal lattice, Lloyd-Max quantizer, Gaussian mixture model (GMM), Polar quantization, Elliptical distribution.

\section{INTRODUCTION}

Quantization is a critical technique for analog-todigital conversion and signal compression [1]. Scalar quantization is simple, fast and easily amenable to a hardware implementation, while vector quantization [2] in high dimension could achieve smaller mean square error (MSE) and better Rate-Distortion (R-D) performance, by jointly considering all the dimensions [3], but at the cost of exponentially increasing quantizer design time and more quantization computations, i.e. at the cost of more codebook design and lookup time.

To reduce the cost, a lot of research has focused on two-dimensional random variables, especially those of circular Gaussian distributions, since circular distributions [4], [5] have a lot of elegant closed-form expressions. The earliest work could be referred to Huang and

The authors are with Department of Electrical and Computer Engineering, University of Florida, Gainesville, FL 32611. Email of Lei Yang: yangalalei@gmail.com.
Schultheiss's method [6], which quantizes each dimension of random variables in Gaussian distributions with separate one-dimensional Lloyd-Max quantizers [7]. It is efficient and effective, but definitely could be improved. Later, Zador [8] and Gersho [9] studied quantization by using companders with a large number of quantization levels theoretically. They used a compressor to transform the data into a uniform distribution, and then applied the optimal quantizers for the uniform distribution, and then transform the data with an expander. But this scheme does not work well under a small number of quantization levels. Another major method for designing quantizers for circular distributions uses polar coordinates, termed as polar quantization. Polar quantization includes separable magnitude quantization and phase quantization. Uniform polar quantization was studied by Moo [10] with uniform magnitude and phase quantization. The optimal ratio between the number of magnitude quantization levels and the number of phase quantization levels was studied by Pearlman [11] and Bucklew et al. [12], [13], and an MMSE restricted polar quantizer is implemented by using a uniform quantizer for the phase angles and a scaled Lloyd-Max quantizer of Rayleigh distribution for the magnitude. But their MMSE scheme does not consider the center of a circular distribution as a quantization level, thus, its MSE performance is sometimes worse than rectangular quantizers and other lattice quantizers, and it does not work well for elliptical distributions neither. Wilson [14] proposed a series of non-continuous quantization lattices which provide almost the optimal performance among the existing polar quantization. It is a kind of unrestricted polar quantization, but without Dirichlet boundaries. Peter et al. [15] improved Wilson's scheme by replacing arc boundaries with Dirichlet boundaries. He showed the optimal circularly symmetric quantizers for circular Gaussian distributions with a small number of quantization levels.

Most of the previous work focuese on Gaussian distributions, and provide numerical results only for Gaussian distributions. Although Gaussian source is considered as the "worst case" source for data compression, which is instructive to construct a robust quantizer [16], the quantizers for Gaussian distributions are far from the optimal quantizers for other distributions. They did 
not consider the elliptical distributions neither, whose optimal quantizers are obviously different from those for circular distributions. Also, they did not provide a unified framework for arbitrary distributions. Therefore, the optimal quantizers for other distributions such as Laplace distributions, elliptical Gaussian and Laplace distributions need investigation under a unified framework.

To address these problems, we propose a unified quantization system to approach the optimal vector quantizers by using transforms and scalar quantizers. The function of transforms, especially unitary transforms and volumepreserving scaling transforms, on signal entropy and signal distortion is discussed. The optimal decorrelation transform is illustrated which turns an arbitrary memory source into a memoryless source in a mixture distribution model. Then we focus on the scalar quantizer design for memoryless circular and elliptical sources. The tri-axis coordinate system is proposed to determine the quantization lattice, i.e. the positions of quantization levels, inspired by the well-known optimal hexagonal lattice for two-dimensional uniformly distributed signals [17]. It provides a unified framework for both circular and elliptical distributions, and encompasses the polar quantization as a special case. The proposed quantizer is also a kind of adaptive elastic lattice quantizer. We will present the simple design methodology, which utilize the Lloyd-Max quantizers for the corresponding onedimensional distributions. The optimality of this scheme is verified on elliptical/circular Gaussian and Laplace distributions. The methodology description and experiments are focused on the bivariate random variables, and the extension to high dimensional random variables is also discussed.

The advantages of our scheme include the following:

1) It provides an elegant quantization lattice for arbitrary number of quantization levels, especially for prime numbers.

2) It almost always has smaller MSE than the other quantizers.

3) It considers both memoryless and memory sources of arbitrary distributions, which include circular distributions, elliptical distributions and mixed distributions.

4) It is in a unified framework of a tri-axis coordinate system.

5) It has small design and implementation complexity.

The rest of the paper is organized as follows. Section II presents the preliminaries of our proposed quantizer. Section III describes the system architecture of transform plus scalar quantization to approximate the optimal vector quantizer. The preprocessing with transforms is discussed in Section IV to decorrelate signals. In Sec- tion $\mathrm{V}$, we present a tri-axis coordinate system, and the methodology to design the optimal scalar quantizer for both circular and elliptical distributions in detail. Experimental results are shown in Section VI. Finally, Section VII concludes the paper.

\section{PRELIMINARIES}

\section{A. n-dimensional MMSE Quantizer and Scaling Law}

Usually, there are three tools to evaluate the performance of a quantizer. Firstly, mean square error (MSE) between input signal $X$ and the reconstructed signal $\hat{X}$, where $X, \hat{X} \in \Re^{n}$, is considered as following,

$$
\mathrm{MSE}=E\left[(X-\hat{X})^{2}\right]
$$

Signal-to-Noise ratio is another evaluation tool.

$$
\mathrm{SNR}=\frac{|\Sigma|}{\mathrm{MSE}}
$$

where $\Sigma$ is the covariance matrix of $X$, and $|\cdot|$ is the matrix determinant operator. The rate-distortion curve is the third one.

Lloyd-Max quantizer [2], [7] is an MMSE quantizer. For one-dimensional signals, let $t_{k}(k=0, \cdots, N)$ denote boundary points of quantization intervals, and let $r_{k}(k=0, \cdots, N-1)$ denote quantization levels. Then Lloyd-Max quantizer is characterized by:

$$
\begin{aligned}
\left\{t_{k}^{*}, r_{k}^{*}\right\} & =\arg \min _{\left\{t_{k}, r_{k}\right\}} \operatorname{MSE} \\
& =\arg \min _{\left\{t_{k}, r_{k}\right\}} \sum_{k=0}^{N-1} \int_{t_{k}}^{t_{k+1}}\left(x-r_{k}\right)^{2} f_{X}(x) d x
\end{aligned}
$$

where $f_{X}(x)$ is the probability density function (pdf) of $X, N$ is the number of quantization levels. From (3), we have the centroid condition, and the nearest neighbor condition:

$$
t_{k}^{*}=\frac{r_{k-1}^{*}+r_{k}^{*}}{2}, \quad k=1, \cdots, N-1,
$$

and

$$
r_{k}^{*}=\frac{\int_{t_{k}^{*}}^{t_{k+1}^{*}} x p(x) d x}{\int_{t_{k}^{*}}^{t_{k+1}^{*}} p(x) d x}, \quad k=0, \cdots, N-1,
$$

$\left[t_{0}^{*}, t_{N}^{*}\right]$ is the range of the input of quantizers.

The Lloyd-Max quantizer for one-dimensional Gaussian distribution with zero mean and unit variance has been well studied. Given the number of quantization levels $N$, the Lloyd-Max quantizer for zero mean, unit variance Gaussian is shown in the tables in [17]. Given the Lloyd-Max quantizer for zero mean, unit variance Gaussian, we can use the affine law in Proposition 1 to obtain the Lloyd-Max quantizer for Gaussian distribution with arbitrary mean $\mu$ and arbitrary variance $\sigma^{2}$. 
Proposition 1: (Affine Law) For a random $X$ with zero mean, assume that its $N$-level Lloyd-Max quantizer is specified by $t_{k}(k=0, \cdots, N)$ and $r_{k}(k=$ $0, \cdots, N-1)$. Then for random $Y=\Sigma^{-\frac{1}{2}} X+\mu$, with mean $\mu$ and covariance matrix $\Sigma$, where $\Sigma=c \cdot I$, $c>0$, its Lloyd-Max quantizer is specified by $\hat{t}_{k}=$ $\Sigma^{-\frac{1}{2}} t_{k}+\mu(k=0, \cdots, N)$ and $\hat{r}_{k}=\Sigma^{-\frac{1}{2}} r_{k}+\mu$ $(k=0, \cdots, N-1)$.

Proof: For one-dimensional random variables, $\Sigma=$ $\sigma^{-2}$, the Lloyd-Max quantizer is:

$$
\min _{r_{k}^{\prime}, t_{k}^{\prime}} \sum_{k=1}^{N} \int_{t_{k-1}^{\prime}}^{t_{k}^{\prime}}\left(x-r_{k}^{\prime}\right)^{2} f_{X}(x) d x
$$

with $\left\{t_{k}\right\}_{k=0}^{N}$ and $\left\{r_{k}\right\}_{k=1}^{N}$ as the solution.

For $Y=\sigma X+\mu$,

$$
\begin{aligned}
& \min _{r_{k}^{\prime \prime}, t_{k}^{\prime \prime}} \sum_{k=1}^{N} \int_{t_{k-1}^{\prime \prime}}^{t_{k}^{\prime \prime}}\left(y-r_{k}^{\prime \prime}\right)^{2} f_{Y}(y) d y \\
& =\sum_{k=1}^{N} \int_{\frac{t_{k-1}^{\prime \prime}-\mu}{\sigma}}^{\frac{t_{k}^{\prime \prime}-\mu}{\sigma}}\left(\sigma x+\mu-r_{k}^{\prime \prime}\right)^{2} f_{X}(x) d x \\
& =\sigma^{2} \sum_{k=1}^{N} \int_{\frac{t_{k-1}^{\prime \prime}-\mu}{\sigma}}^{\frac{t_{k}^{\prime \prime}-\mu}{\sigma}}\left(x-\frac{r_{k}^{\prime \prime}-\mu}{\sigma}\right)^{2} f_{X}(x) d x
\end{aligned}
$$

if and only if $\frac{t_{k-1}^{\prime \prime}-\mu}{\sigma}=t_{k}$ and $\frac{r_{k}^{\prime \prime}-\mu}{\sigma}=r_{k}$, Eq. (7) is minimal, i.e. $\left\{\sigma t_{k}+\mu\right\}_{k=0}^{N}$ and $\left\{\sigma r_{k}+\mu\right\}_{k=1}^{N}$ is the solution for Eq. (7).

Similarly, it holds for $n$-dimensional random variables.

It indicates that for a random variable obtained from another random variable by an affine transform, the optimal quantizer could be obtained from the original quantizer by the same affine transform. In the following, we focus our investigation on random variables with zero means and diagonal covariance matrices.

\section{B. Circular and Elliptical Distributions}

The source of a circular distribution is a memoryless source. Each dimension of data is of exactly the same one-dimensional distribution. For example, a twodimensional circular Gaussian distribution with unitary variance is shown as following:

$$
f\left(x_{1}, x_{2}\right)=\frac{1}{2 \pi} e^{-\frac{x_{1}^{2}+x_{2}^{2}}{2}}
$$

It could also be represented in polar coordinate system as following:

$$
f(r, \phi)=\frac{1}{2 \pi} r e^{-\frac{r^{2}}{2}}
$$

That is why Lloyd-Max quantizer for Rayleigh distribution is preferred to quantize signal magnitudes in restricted polar quantization.
The source of an elliptical distribution could be memoryless source with erect principal axes, and memory source with skewed principal axes. Memory source could be decorrelated with unitary transforms into memoryless source whose component in each dimension is of the same distribution but with possible different variances. The memoryless elliptical Gaussian source could be represented as follows:

$$
f\left(x_{1}, x_{2}\right)=\frac{1}{2 \pi \sigma_{1} \sigma_{2}} e^{-\frac{\left(x_{1}-\mu_{1}\right)^{2}}{2 \sigma_{1}^{2}}} \cdot e^{-\frac{\left(x_{2}-\mu_{2}\right)^{2}}{2 \sigma_{2}^{2}}}
$$

Uniform distribution is both an ordinary circular distribution and an ordinary elliptical distribution.

\section{Ideal Uniform Distribution and Optimal Two- dimensional Hexagonal Lattice}

Vector quantization aims to minimize MSE, but its computational complexity is really high and increases exponentially with the number of quantization levels. For uniform distribution, we generally accept that the optimal VQs are regular voxels in the source domain. Whereas, due to the finite domain constrain, regular voxels cannot just pad the space by exactly integer number of voxels. Therefore, the VQs of uniform distributions found by various algorithms compose of irregular voxels, which are degenerated from the regular ones. We build our theorem on the infinite domain, i.e. infinite dynamic range, to avoid boundary dilemma. Thus, we define ideal uniform distribution as follows.

\section{Definition 1: Ideal Uniform Distribution}

Given domain $V$ of distribution whose volume is $\Omega(V)$, the uniform distribution is

$$
f(x)=\left\{\begin{aligned}
\frac{1}{\Omega(V)}, & x \in V \\
0, & o / w .
\end{aligned}\right.
$$

When $\Omega(V) \rightarrow \infty$, then $f(x)$ is an ideal uniform distribution. There is no boundary constraint for optimal VQ designing in ideal uniform distributions.

Lemma 1: The optimal VQ for the two-dimensional ideal uniform distribution is regular honeycomb as shown in Fig. 5.

\section{SyStEM ARCHITECTURE}

\section{A. Quantization for Compression}

The general coding system usually includes transform, quantization and entropy coding as shown in Fig. 1. The optimal transform could simplify vector quantization scheme into scalar quantization, and even replace variable-length entropy coding in the coding system with fixed length coding. Rate-Distortion code is an optimal code proposed by Shannon [3]. It is an optimal vector code when block length $n \rightarrow \infty$. Only is known its 
existence, but not its design in a general case. Vector quantization has the ability to approach Rate-Distortion bound when number of quantization levels $N \rightarrow \infty$, but is overwhelmed by the exponentially increasing complexity. Therefore, vector quantization is desired to be replaced by transform followed by scalar quantization with the same Rate-Distortion performance but much less design and implementation complexity, as adopted by a general transform compression system shown in Fig. 1. Therefore, an optimal transform plus an optimal scalar quantizer gives us a new promising guideline to achieve Rate-Distortion bound as presented in the next Sections.

\section{B. Theorem and System Framework}

Therefore, we give a theorem as follows:

Theorem 1: The MMSE vector quantization could be achieved by transformation followed by scalar quantization.

It will be proved in Section $\mathrm{V}$ for uniform distributions.

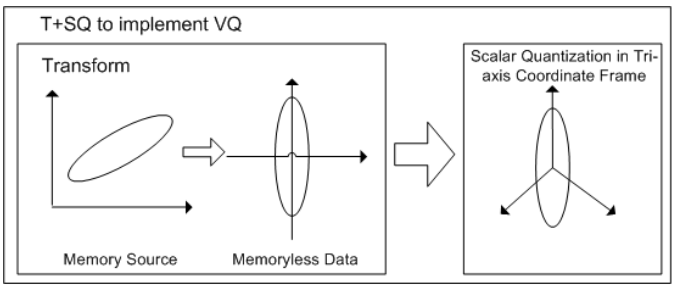

Fig. 2. System architecture to implement VQ with a transform plus scalar quantization.

Following Theorem 1, we propose a system architecture as shown in Fig. 2. A vector quantizer is implemented by a transformation and a scalar quantizer. The transformation we focus on can be a linear transform with high decorrelation ability. We will discuss the unitary transforms, volume-preserving scaling transforms and the optimal decorrelation transforms in Section IV. The scalar quantizer is implemented in the tri-axis coordinate system which will be described in detail in Section V. The transformation plus scalar quantization has the advantage of possible small complexity and good R-D performance. The system still has issue of tradeoff between complexity $(\mathrm{C})$, rate $(\mathrm{R})$ and distortion (D). Therefore, the system design should comprise the C-R$\mathrm{D}$ theory. Best R-D performance with least complexity is desired.

The proposed system is flexible, in which the companding technique could also be plugged in as shown in Fig. 3. As we will show later, the companding technique is asymptotically optimal, but may not work well in low rate situation. But our method with the tri-axis coordinate system works almost universally.

\section{PREPROCESSING WITH TRANSFORMS}

Transform is helpful for quantization. Any mapping is a transform. Nonlinear transforms introduce undesired nonlinear error after quantization. Therefore, linear transform is considered in this section. To preserve signal energy constant, linear transforms, such as unitary transforms and volume-preserving scaling transforms, represented by matrices with unitary determinant, are focused on.

\section{A. Unitary Transforms}

Unitary Transforms are rotations or rotations with reflection in Euclidean space, aiming at high decorrelation ability.

We know that signal-dependent Karhunen-Loeve transform (KLT) is optimal in the sense of the highest decorrelation ability for finite block length signals. While DCT is a fixed transform and a good substitution of KLT.

Lemma 2: Mean Square Error is invariant under unitary transforms.

Proof: A unitary transform $Q$ satisfies $Q^{*} Q=$ $Q Q^{*}=I$. For vectors $x$ and $y$, the Euclidean distance between them is $\|x-y\|$. After transform by $Q$, the Euclidean distance between $Q x$ and $Q y$ is $\|Q x-Q y\|^{2}=$ $\|Q \cdot(x-y)\|^{2}=(x-y)^{*} \cdot Q^{*} \cdot Q \cdot(x-y)=\|x-y\|^{2}$. Thus, Mean Square Error is invariant under unitary transforms.

Lemma 3: The MMSE vector quantizers of random vectors after unitary transformation are the MMSE vector quantizers of the random vectors after the same unitary transformation.

Lemma 3 is easy to obtain from Lemma 2, and is a rotation-invariant property of MMSE vector quantizers.

Lemma 4: The sum of entropy of each component of random vector $X$ will decrease after decorrelation by unitary transforms.

Proof: Denote the components of random vector $X$ as $\left(X_{1}, X_{2}\right)$ and a unitary transform as $U$. After transform, the random vector becomes $X^{\prime}=\left(X_{1}^{\prime}, X_{2}^{\prime}\right)$. Neglect the precision loss brought by the computer, $U$ is a one-to-one mapping. Therefore, $H\left(X_{1}, X_{2}\right)=$ $H\left(X_{1}^{\prime}, X_{2}^{\prime}\right)$. If $X_{1}^{\prime}$ and $X_{2}^{\prime}$ are independent, then $H\left(X_{1}^{\prime}\right)+H\left(X_{2}^{\prime}\right)=H\left(X_{1}^{\prime}, X_{2}^{\prime}\right)=H\left(X_{1}, X_{2}\right) \leq$ $H\left(X_{1}\right)+H\left(X_{2}\right)$

\section{B. Scaling Transforms}

From Affine Law in Proposition 1, we know that the MMSE quantizers will undergo the similar expansion or shrinkage as input signals, whose dimensions are scaled 
by a same factor. This kind of transforms could be represented as the following matrix:

$$
c \cdot\left(\begin{array}{cccc}
a & 0 & \cdots & 0 \\
0 & a & \cdots & 0 \\
\vdots & & \ddots & \vdots \\
0 & \cdots & 0 & a
\end{array}\right)
$$

It will increase or decrease signal volume uniformly.

Thus, we steer to Volume-preserving scaling transform.

Definition 2: Volume-preserving scaling transform could be represented by a diagonal matrix with unitary determinant, i.e., the product of all the diagonal elements of the diagonal matrix is 1 as following:

$$
\left(\begin{array}{cccc}
a_{1} & 0 & \cdots & 0 \\
0 & a_{2} & \cdots & 0 \\
\vdots & & \ddots & \vdots \\
0 & \ldots & 0 & a_{n}
\end{array}\right)
$$

where $\prod_{i=1}^{n} a_{i}=1$.

Usually the MSE and energy of signals change after volume-preserving scaling transformation, since $\sum_{i=1}^{n} x_{i}^{2} \neq \sum_{i=1}^{n} a_{i}^{2} x_{i}^{2}$. The rate-distortion theory requires the MSE uniformly distributed among each component of the random vector, if the MSE does not exceed the variance of that component. Therefore, the MMSE vector quantizer for an elliptical distribution could not be obtained from the MMSE vector quantizer for a circular distribution by a simple scaling. That is why the existing works seldom consider elliptical distributions, or independent consider the circular and elliptical distributions. But they are unified in our system.

Lemma 5: The sum of the entropy of every component of a random vector in Gaussian or Laplace distribution keeps constant after volume-preserving scaling transform.

Proof: Denote the variance of every component of an $n$-dimensional Gaussian vector $X=$ $\left(X_{1}, X_{2}, \cdots, X_{n}\right)$ as $\left(\sigma_{1}^{2}, \sigma_{2}^{2}, \cdots, \sigma_{n}^{2}\right)$. After volumepreserving scaling transforms, the resulted Gaussian vector $X^{\prime}$ has variances of $\left({\sigma_{1}^{2}}^{\prime}, \sigma_{2}^{2 \prime}, \cdots, \sigma_{n}^{2^{\prime}}\right)$ for each component and $\prod_{i=1}^{n} \sigma_{i}^{2}=\prod_{i=1}^{n}{\sigma_{i}^{2}}^{\prime}$. From information theory, we know that entropy of Gaussian is: $H\left(X_{i}\right)=\frac{1}{2} \ln \left(2 \pi e \sigma_{i}^{2}\right), i=1, \cdots, n$. Therefore, $\sum_{i=1}^{n} H\left(X_{i}\right)=\sum_{i=1}^{n} \frac{1}{2} \ln \left(2 \pi e \sigma_{i}^{2}\right)=$ $\frac{1}{2} \ln \left((2 \pi e)^{n} \prod_{i=1}^{n} \sigma_{i}^{2}\right)=\frac{1}{2} \ln \left((2 \pi e)^{n} \prod_{i=1}^{n} \sigma_{i}^{2^{\prime}}\right)=$ $\sum_{i=1}^{n} H\left(X_{i}^{\prime}\right)$

Similarly, it holds for random vectors of Laplace distribution.

\section{Transforms with Unitary Determinant}

A transform matrix with unitary determinant is a volume-preserving transform. A general volume- preserving transform may change both sum of subband entropy and MSE.

A matrix $A$ has a QR factorization: $A=Q R$, where $Q$ is an unitary matrix and $R$ is an upper triangular matrix. The diagonal elements of $\mathrm{R}$ could be extracted to make a diagonal matrix $D: R=U D$, where $U$ is unit upper triangular, and $D$ is diagonal. Then for $A$ with unitary diterminant, we can have a factorization: $A=Q U D$, where $Q$ is unitary or rotation, $U$ is unit upper triangular or shearing, and $D$ is volume-preserving scaling transform. Besides that unitary transforms change sum of subband entropy and that volume-preserving scaling transforms change MSE, shearing will change both sum of subband entropy and MSE.

\section{Optimal Transform for Arbitrary Distributions}

Since the volume-preserving scaling transforms perturb the MSE of each component, we only consider unitary transforms before quantization for MMSE quantizers.

If an arbitrary distribution is considered, the most powerful decorrelation might not be achieved by a simple unitary transform, since unitary transforms generally can not decrease both the intra-component/block correlation and the inter-component/block correlation.

An arbitrary distribution can be approximated by a Gaussian Mixture Model (GMM) found by expectation maximization (EM) algorithm. The pdf of a GMM random variable $X$ is given below:

$$
f_{X}(x)=\sum_{i=1}^{N_{g}} p_{i} \cdot g_{i}(x)
$$

where $N_{g}$ is the number of Gaussian components in the GMM; $g_{i}(x)$ is the Gaussian pdf for component $i\left(i=1, \cdots, N_{g}\right)$ shown as an ellipse in Fig. 4; $p_{i}$ denotes the probability of component $i\left(i=1, \cdots, N_{g}\right)$; and $\sum_{i=1}^{N_{g}} p_{i}=1$. Assume the data come from the neighbor pixel pairs in a gray image. Then Gaussian components of data almost fall along the diagonal due to the correlation between pixel pairs. For decorrelation, the first step is for intra-component decorrelation, the second step is for inter-component decorrelation. The two steps could change order. Later the scalar quantizers could be applied to each decorrelated Gaussian component.

\section{OPTIMAL SCALAR QUANTIZERS IN TRI-AXIS COORDINATE SYSTEM}

After transformation, we can obtain random vectors with independent components. For Theorem 1, onedimensional vector quantization is scalar quantization. No transform is needed. It is a trivial case. For twodimensional vector quantization, we will prove this theorem for uniform distributions in a tri-axis coordinate 
system. For high dimensional vector quantization, multiaxis coordinate system is needed.

\section{A. Tri-axis Coordinate System}

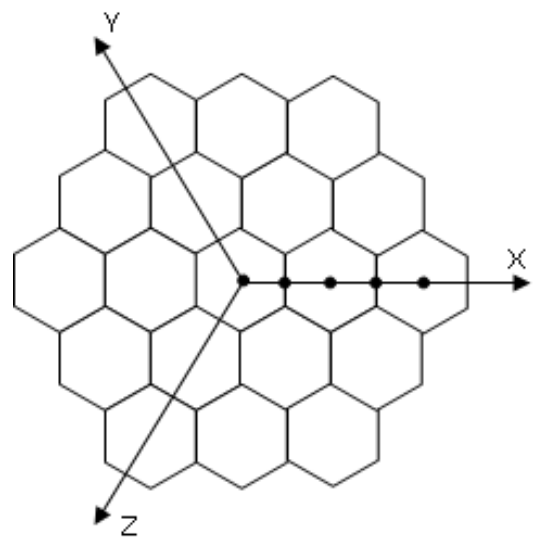

Fig. 5. Two dimensional tri-axis coordinate system.

Definition 3: The tri-axis coordinate system in a twodimensional space has three axes $X, Y$ and $Z$, and the angles are $120^{\circ}$ between the three axes $X, Y$ and $Z$.

The tri-axis coordinate system is shown in Fig. 5. Every point in the two-dimensional space can be represented as a point in this tri-axis coordinate system.

\section{B. Orthonormal Property of Tri-Axis Coordinate System}

Proposition 2: Any point in the tri-axis coordinate system $(x, y, z)$ satisfies $x+y+z=0$.

Proof: Unit vectors along three axes $X, Y$ and $Z$ could be $\vec{X}=(1,0)^{T}, \vec{Y}=(-1 / 2, \sqrt{3} / 2)^{T}$ and $\vec{Z}=(-1 / 2,-\sqrt{3} / 2)^{T}$. A point $P=(u, v)$ in Cartesian coordinate system could be represented by $(x, y, z)$ in tri-axis coordinate system with $x=P \cdot \vec{X}, y=P \cdot \vec{Y}$ and $z=P \cdot \vec{Z}$. Then $x+y+z=P \cdot \vec{X}+P \cdot \vec{Y}+P \cdot \vec{Z}=$ $P \cdot(\vec{X}+\vec{Y}+\vec{Z})=P \cdot 0=0$.

The big advantage of the tri-axis system is that the coordinate lattice is hexagonal, which is what we need for optimal vector quantization, while bi-axis systems can only give quadrilateral coordinate lattices. Linear transforms preserve linearity and parallelism, therefore, if a linear transform is applied, the coordinate lattice is still hexagonal and with three pairs of parallel edges although the angles are not neccessary $120^{\circ}$. The optimal vector quantizer for a uniform distribution is perfect represented by the tri-axis coordinate system as shown in Proposition 3, but not by polar coordinate system.
The coordinates $(x, y, z)$ are highly correlajted. For some symmetrical distributions, two axes or one axis is sufficient. For example, the optimal two-dimensional vector quantization for uniform distributions could be determined by two axes $X, Y$ of $120^{\circ}$ in spanning a hexagonal lattice. The optimal two-dimensional vector quantization for circular distributions also needs two axes, one of which determines the magnitude quantization, another one determines the phase quantization. It is rotation-invariant for circular distributions, but not for elliptical distributions. We will show them in next subsections.

\section{Tri-Axis Coordinate System for Uniform Distribution}

Fig. 6. Two dimensional optimal uniform vector quantizer.

It is well known that the optimal vector quantizer for uniform distributions in a two-dimensional space is regular honeycomb [17], which is from the geometry of numbers, also from discrete geometry in the Euclidean space. We will implement it with scalar quantization in tri-axis system as shown in Fig. 6.

Proposition 3: Hexagonal lattice in tri-axis system is still Rate-Distortion optimal for quantization of uniform distributions.

Proof:

1) Vector quantization levels are the centroids of the hexagons. The centroid of each hexagon of the optimal quantizer could be represented by a fixed length code. $R=\log _{2} N$, where $N$ is the number of quantization levels.

2) Every point in the two-dimensional space could be represented by the vector $\vec{r}=c_{1} \vec{r}_{1}+c_{2} \vec{r}_{2}$, as shown in Fig. 6, where $\vec{r}_{1}$ and $\vec{r}_{2}$ are the basis vectors of VQ. $c_{1}$ and $c_{2}$ are integers and uniformly distributed if the centroid is uniformly distributed. 
3) Scalar quantizers compose of two independent scalar quantizers along two axes $\vec{r}_{1}{ }^{\prime}$ and $\vec{r}_{2}{ }^{\prime}$. Every point could be represented by two indices in the codebook. The indices are obtained by projecting the point to the nearest code on the axes. For example, a point representation is $\vec{r}=x \vec{r}_{1}^{\prime}+y \vec{r}_{2}{ }^{\prime}$ which is quantized to $\vec{r}=x_{m} \vec{r}_{1}^{\prime}+y_{n} \vec{r}_{2}^{\prime}$.

4) Let $\vec{r}_{1}=\vec{r}_{1}{ }^{\prime}$ and $\vec{r}_{2}=\vec{r}_{2}{ }^{\prime}$, then we have $c_{1}=x_{m}$ and $c_{2}=y_{m}$. The sum of square error of SQ is the inner product of $\left(x-x_{m}\right) \vec{r}_{1}{ }^{\prime}$ and $\left(y-y_{m}\right) \vec{r}_{2}{ }^{\prime}$, and the sum of square error of VQ is the inner products of $\left(x-c_{1}\right) \vec{r}_{1}$ and $\left(y-c_{2}\right) \vec{r}_{2}$. Thus, VQ and SQ have the same distortion.

5) For uniform distribution, only one codebook is needed for two axes $\vec{r}_{1}{ }^{\prime}$ and $\vec{r}_{2}{ }^{\prime}$. The indices could be coded with a fixed length. $R_{i}=\log _{2} N_{i}$, where $N_{i}$ is the number of quantization levels along axis $i(i=1,2)$.

6) $N_{1} \times N_{2}=N$ asymptotically, i.e. $R_{1}+R_{2}=$ $\log _{2} N_{1}+\log _{2} N_{2}=\log _{2} N=R$, VQ and SQ have the same rate.

7) Therefore, SQ and VQ are with the same R-D performance.

Therefore, the optimal MMSE vector quantization could be achieved by a transform (an identical transform) followed by a uniform scalar quantizer for a twodimensional ideal uniform distribution.

In this way, SQ and VQ have the same R-D performance, while the codebook size of SQ is around the square root of that of VQ. Because of the reduced complexity provided by $\mathrm{SQ}$, we try to use $\mathrm{SQ}$ to replace $\mathrm{VQ}$ in this paper. Another point needed to mention is that the numbers of quantization levels for hexagon lattice are prime numbers $1,7,19,37, \cdots$, growing along circles with larger and larger radius, which could be found in Fig. 5. Therefore, it is easy to design quantizers of prime number of quantization levels with hexagonal lattices, while it is difficult and inferior with rectangular lattices for circular distributions.

\section{Tri-Axis Coordinate System for Circular and Ellipti- cal Distributions}

How about the distribution is not uniform, what will the optimal quantization lattices be? This is a problem of finding the transform from a non-uniform distribution to a uniform distribution.

1) Elastic Quantization Lattices for Circular and Elliptical Distributions: From the optimal hexagonal lattice for a uniform distribution, we state that the optimal vector quantizer for a circular distribution forms an expanded hexagonal lattice, as shown in Fig. 7. The expansion ratio between the optimal lattice for a two-dimensional circular distribution and that of a two-dimensional uniform distribution along the radius

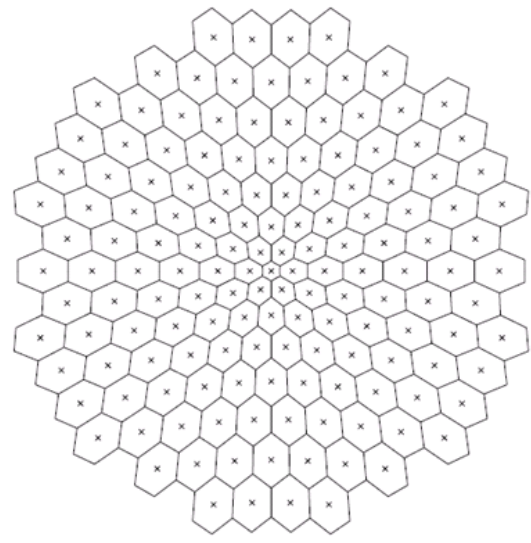

Fig. 7. Circularly expanded hexagon lattice for two-dimensional circular Gaussian distribution.
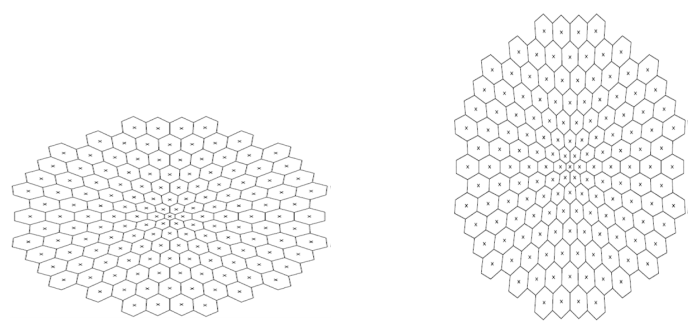

(a) Horizontal elliptical hexagonal lat- (b) Vertical elliptical hexagotice nal lattice

Fig. 8. Elliptically expanded hexagon lattice for two-dimensional Elliptical Gaussian distribution.

direction may approximately follow the expansion ratio between the Lloyd-Max quantizer for the corresponding one-dimensional distribution and that of a onedimensional uniform distribution, i.e. the Lloyd-Max quantizer for the corresponding one-dimensional distribution.

2) Design Methodology: We firstly focus on the positions of quantization levels of a two-dimensional vector quantizer. The lattice patterns of the proposed quantizer are determined beforehand, as shown in Fig. 10 and Fig. 11. The quantization levels approximately fall on the centroids of the lattice, which are uniformly distributed in each annulus. We restrict them to be in the same circle for simplicity, and each quantization region of lattice do not need to be hexagonal. In different annuluses, quantization levels are staggered arranged similar to those of the rotated polar quantization [15]. The optimal distance between the quantization levels and the origin for the magnitude quantization is determined by weighting the Lloyd-Max quantizer of the corresponding onedimensional distribution with the unitary-variance. For 
more precise locations of MMSE magnitude quantization levels, they are further searched outwards in radial directions for MMSE.

To be specific, for a two-dimensional circular distribution, its pdf could be separately represented in the polar coordinate system as $f(r, \theta)=f_{1}(r) \cdot f_{2}(\theta)$, while it is not for elliptical distributions. For an arbitrary elliptical distribution, the data could be transformed by unitary transforms into a distribution whose principal axes are parallel to the coordinate systems, and then shifted to the origin. After such transformation, the equal-probability contours of distributions could be uniformly represented by the following equation in Cartesian coordinate system:

$$
\sum_{i=1}^{n} \frac{x_{i}^{2}}{b_{i}^{2}}=1
$$

$b_{1}=b_{2}=\cdots=b_{n}=b$ for circular distributions; $b_{i}$ s are not all equal for elliptical distributions. The weighting effect from $b_{1}$ and $b_{2}$ for elliptical distributions is important. Because if the quantizers of circular distributions is used for elliptical distributions, the resultant MSE per each dimension has ratio of $\frac{b_{1}^{2}}{b_{2}^{2}}$. Whereas, from Shannon Rate-Distortion theory (i.e. reverse water filing), we know that the if the MSE is less than the variance of each component, bit-rate should be allocated such that the MSE per dimension is nearly equal. Therefore, we should use $b_{1}$ and $b_{2}$ to weight quantization levels towards this for elliptical distributions.

The magnitude quantization is non-uniform. For both circular and elliptical distributions, the two-dimensional quantization levels fall on each circle or oval could be represented by the coordinates $\left(c \cdot b_{1} \cdot \cos \theta, c \cdot b_{2} \cdot \sin \theta\right)$ shown as stars in Fig. 9. $c$ increases non-uniformly in radial directions. $c$ could be determined by searching outward starting from Lloyd-Max quantization for Gaussian distribution in radial directions.

The uniform phase quantization is optimal for circular distributions, but may not for elliptical distributions. We take uniform phase quantization for both kinds of distributions, since the optimal phase quantization for elliptical distributions is a little perturbation from the uniform phase quantization. We will show its suboptimality for elliptical distribution in experiments. As shown in Fig. 10, the number of quantization levels in each annulus is $1,6,12,18$, similar to that of the regular hexagonal lattice. Within each magnitude annulus, the $k$ phase regions all have equal size, whose boundaries are represented as follows:

$$
(j-1) \frac{2 \pi}{k} \leq \theta<j \frac{2 \pi}{k}
$$

where $j=1,2, \cdots, k$.

The boundaries of quantization intervals are obtained by the nearest neighborhood scheme based on the fixed

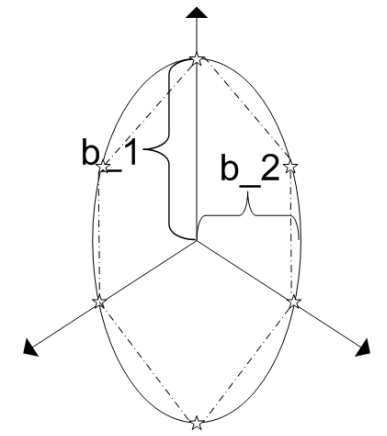

Fig. 9. Tri-axis frame for a general two-dimensional elliptical distribution.

quantization levels as shown in Eq. (4). The resultant quantization regions are not necessarily hexagonal. The optimal VQ for any distributions, including uniform distributions, in finite regions is deformed from hexagonal lattices in this way.

3) The Number of Quantization Levels in Each Annulus: How many quantization levels should we assign to each annulus? Previously, for the restricted polar quantization [11], quantization levels $N$ is factorized into $N=N_{\theta} \cdot N_{r}$, where $N_{\theta}$ is the number of quantization levels in each annulus, and $N_{r}$ is the number of annuluses. Although the optimal ratio between $N_{\theta}$ and $N_{r}$ is studied, some numbers of $N$ can not be perfectly factorized, not to mention a prime number. This difficulty also lies in the unrestricted polar quantization [14]. The non-continuity of quantization patterns exists in all the previous works. It is also an imperfection in our schemes. We have two schemes to arrange magnitude quantization levels vs. phase quantization levels. Our quantizer design and optimization methodology is much simpler than that of the unrestricted polar quantization.

The first scheme allows freedom in the number of phases assigned at each magnitude level. The optimal patterns are derived from experiments, which are coincident with Wilson's scheme [14] but with better performance and Dirichlet boundaries, as shown in Fig. 12.

The second one is the progressive quantization scheme [18] as shown in Fig. 13. The number of annuluses $L$ increases with the number of quantization levels $N=1,7,19, \cdots, 1+6 \cdot(1+2+3+\cdots)$. Define set $N_{L}=\{1,7,19, \cdots, 1+3 l(l+1)\}$ and $N_{L}(l)$ is the $l$-th element in set $N_{L}$. That is the number of annuluses $L$ is determined by:

$$
L=\left\{\begin{aligned}
\inf \left\{l: N-3 l \leq N_{L}(l)\right\}, & N \leq 4 \\
\inf \left\{l: N+7-6 l \leq N_{L}(l)\right\}, & o / w
\end{aligned}\right.
$$

where inf is the infimum. Therefore, the quantizer 
could be implemented progressively with the increase of $N$. The previously located quantization levels need not change their relative positions, only their magnitudes should be shrinked a little as suggested by the Lloyd-Max quantizer of one-dimensional Gaussian. Or hierarchically, we could further quantize each existing quantization region with our scheme.

Comparing the two schemes of quantization lattice patterns, we can see that the quantitative descriptions of the first optimal scheme are difficult to provide. For small $N$, the second scheme has performance close to the first scheme, although with possible different lattice patterns; for large $N$, their performance difference decreases, and the quantization patterns of the second scheme is asymptotically approaches those of the first scheme. Scheme one and scheme two have a lot of common quantization lattice patterns.

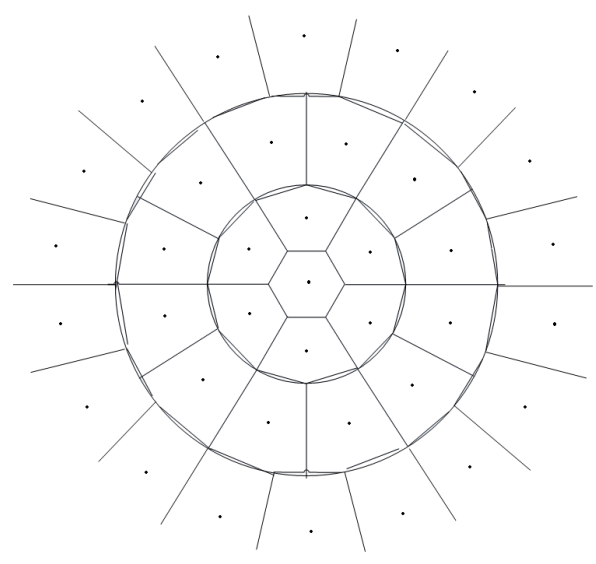

Fig. 10. Expanded hexagonal lattice for two-dimensional circular Gaussian distribution.

4) Expansion Rule: How far away the expansion rule along radial direction for two-dimensional distributions from the Lloyd-Max quantizer for the corresponding one-dimensional distributions? Take Gaussian distribution for example. For two-dimensional Gaussian with joint pdf given by:

$$
\left.P_{X}\left(x_{1}, x_{2}\right)=\frac{1}{2 \pi} \exp \left\{-\frac{x_{1}^{2}+x_{2}^{2}}{2}\right\}\right)
$$

where $-\infty<x_{1}, x_{2}<\infty$. Its polar coordinate representation is:

$$
P_{R, \Theta}(r, \theta)=\frac{r}{2 \pi} \exp \left\{-r^{2} / 2\right\}
$$

where

$$
\begin{gathered}
0 \leq r<\infty, 0 \leq \theta<2 \pi \\
r=\left(x_{1}^{2}+x_{2}^{2}\right)^{1 / 2}
\end{gathered}
$$

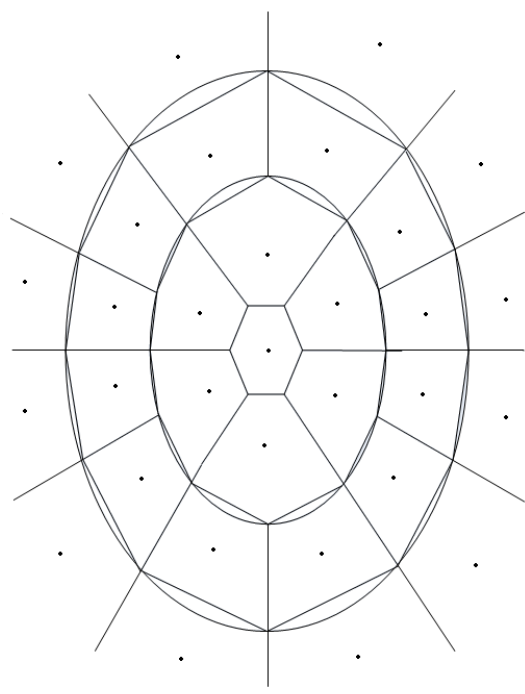

Fig. 11. Expanded hexagonal lattice for two-dimensional elliptical Gaussian distribution.

$$
\theta=\tan ^{-1}\left(\frac{x_{2}}{x_{1}}\right)
$$

The number of annuluses $L$ of the quantizer for two-dimensional circular Gaussian has the following relationship with the number $N_{1}$ of quantization levels of one-dimensional Gaussian distribution.

$$
N_{1}=\left\{\begin{array}{r}
2 L, L=1 \\
2 L-1, L \geq 2
\end{array}\right.
$$

Then the expansion rule for $r$ in Eq. (17) with $L$ annuluses is found in table of the Lloyd-Max quantizer for one-dimensional Gaussian with $N_{1}$ quantization levels. For example, $N=7, L=2$ case as shown in Fig. 12. It corresponds to $N_{1}=3$ of the quantizer for one-dimensional Gaussian, i.e., $r_{1}=0, r_{2}=1.2240$.

Then how far away of $r_{1}=0, r_{2}=1.2240$ from the optimal $r_{1}^{*}, r_{2}^{*}$ ? Consider an upper bound of the difference between $r_{1}$ and $r_{1}^{*}$ when $L=1$. The radial expansion is following the rule for Rayleigh distribution. But there is no quantization level at origin for Rayleigh distribution, so we have to utilize the quantizer for Gaussian distribution for our quantizer.

$$
\frac{\int_{0}^{\infty} \int_{\theta_{0}}^{\theta_{1}} \frac{r^{2}}{2 \pi} e^{-\frac{r^{2}}{2}} d \theta d r}{\int_{0}^{\infty} \int_{\theta_{0}}^{\theta_{1}} \frac{r}{2 \pi} e^{-\frac{r^{2}}{2}} d \theta d r}=\sqrt{\frac{\pi}{2}}
$$

The Lloyd-Max quantizer for one-dimensional Gaussian distribution is as follows:

$$
\frac{\int_{0}^{\infty} \frac{x}{\sqrt{2 \pi}} e^{-\frac{x^{2}}{2}} d x}{\int_{0}^{\infty} \frac{1}{\sqrt{2 \pi}} e^{-\frac{x^{2}}{2}} d x}=\sqrt{\frac{2}{\pi}}
$$

Then the upper bound of the difference is around 0.46 

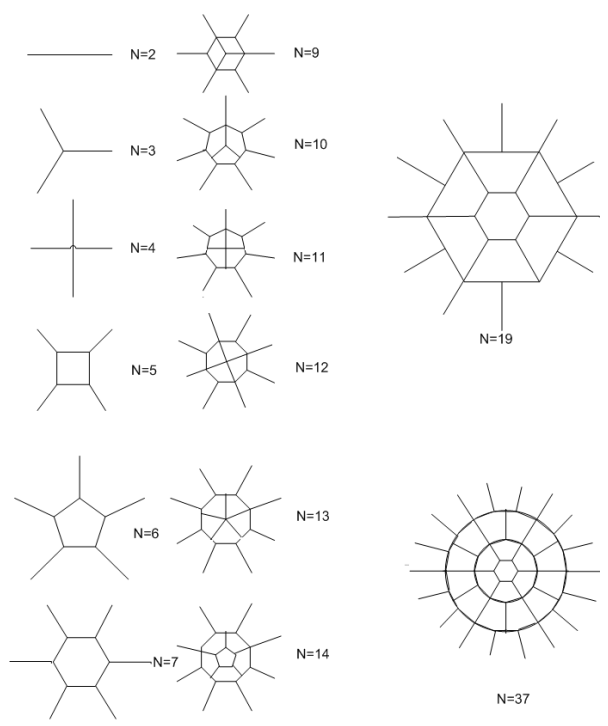

$\mathrm{N}=37$

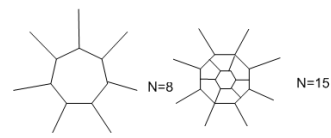

Fig. 12. The first optimal quantization scheme.

$\left(=\sqrt{\frac{\pi}{2}}-\sqrt{\frac{2}{\pi}}\right)$ for uni-variance distributions. As $N$ gets larger, the difference becomes smaller. These are also the maximal searching ranges to find the optimal magnitude quantizers. The Lloyd-Max quantizer for one-dimension Gaussian is a good initial for finding the optimal magnitude quantization for one-dimensional distributions.

The magnitude quantization is almost independent of the phase quantization. It means that when phase quantization changes, the magnitude quantization suffers a little perturbation at most. As the number of quantization levels goes larger, the perturbation turns smaller.

\section{E. Generalization to GMM or LMM}

We can use EM algorithm to identify the components in Gaussian Mixture Model (GMM) or Laplace Mixture Model (LMM). Then for each component, the proposed transformation plus scalar quantizer could be used to replace vector quantizers to approximate the optimal quantization performance.

\section{F. Generalization to High Dimension}

The dodecahedron is the optimal voxel of vector quantization for signals of an ideal three-dimensional uniform distribution [19]. The three-dimensional six-axis coordinate system, composing of six axes for six parallel face pairs of dodecahedron, can be built up similarly as shown in Fig. 14, and its coordinates(a,b,c,d,e,f)

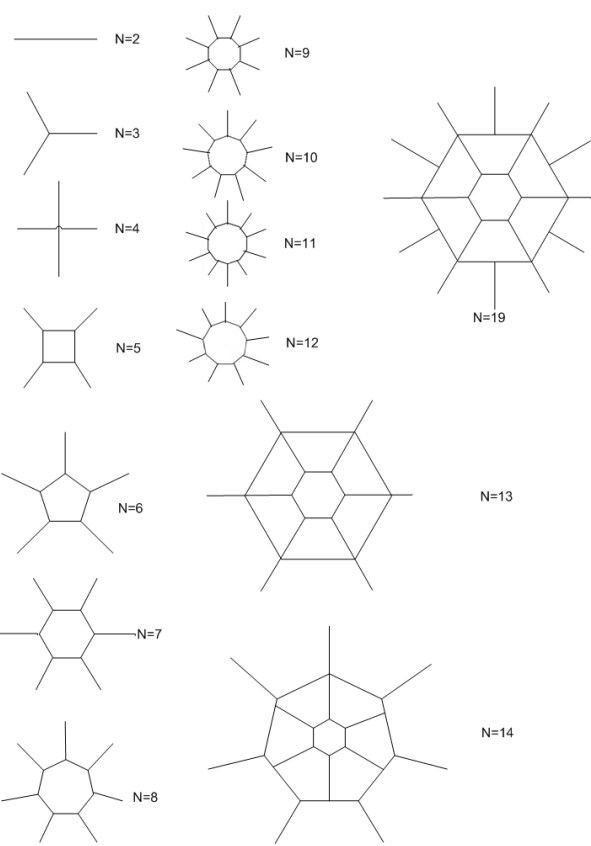

Fig. 13. the second progressive quantization scheme.

have three independent components. Analogously, vector quantization could also be approximated by transformation plus scalar quantization for three-dimensional distributions. For even higher dimensional space, once

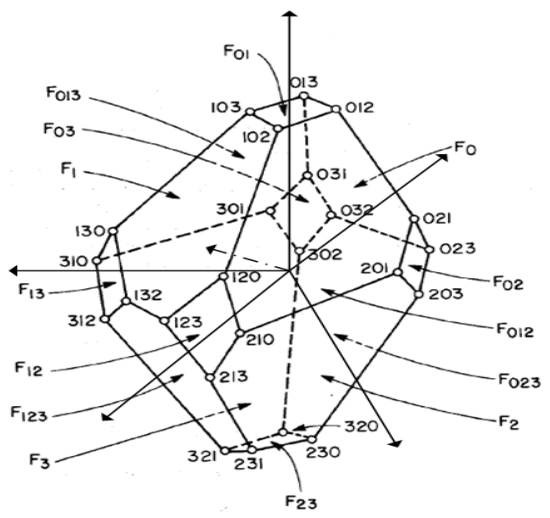

Fig. 14. Hexa-axis coordinate system for quantization of threedimensional distributions.

the optimal voxel of vector quantization for signals of a uniform distribution is obtained, we can obtain the optimal transformation plus scalar quantization to replace vector quantization accordingly. Quantization levels are uniformly located on each sphere. 


\section{EXPERIMENTAL RESUlTS AND Discussions}

In this section, we first show the basic properties of the proposed scalar quantizer. Then we show experimental results with a two dimensional memoryless source of unitary circular Gaussian and Laplace distributions, and elliptical Gaussian and Laplace distributions with $b_{1}=$ $2, b_{2}=1$.

We will compare MSE and the Rate-Distortion performance of our proposed quantizers based on the first scheme with optimal quantization lattices, the unrestricted polar quantizers [14] (indicated by 'UPQ'), the restricted polar quantizers [11] (indicated by 'PQ'), the rectangular quantizers [6] (indicated by 'Rectangular'). The rate here is defined as $\log _{2} N / 2$. The distortion is shown with MSE per dimension. The benchmark is RateDistortion function for a Gaussian memoryless source:

$$
R(D)=\frac{1}{2} \log _{2}\left(\frac{1}{D}\right)
$$

where $0<D \leq 1$. Each quantizer is tested for its best performance, with the corresponding optimal quantization levels, and the optimal ratio between the number of phase quantization levels and the number of magnitude quantization levels. For example, the rectangular quantizers are almost tested with $n^{2}$ quantization levels for circular distributions, i.e. each dimension is quantized by a Lloyd-Max quantizer with $n$ quantization levels, and $2 n \times n$ for elliptical distributions, i.e. data is quantized by a Lloyd-Max quantizer with $2 n$ and $n$ quantization levels respectively applied to the two dimensions. We will not show the results of the vector quantizers found by LBG algorithm, since it is highly initial dependent and the results we obtained are much worse than those of our proposed quantizers.

\section{A. Basic Optimal Properties}

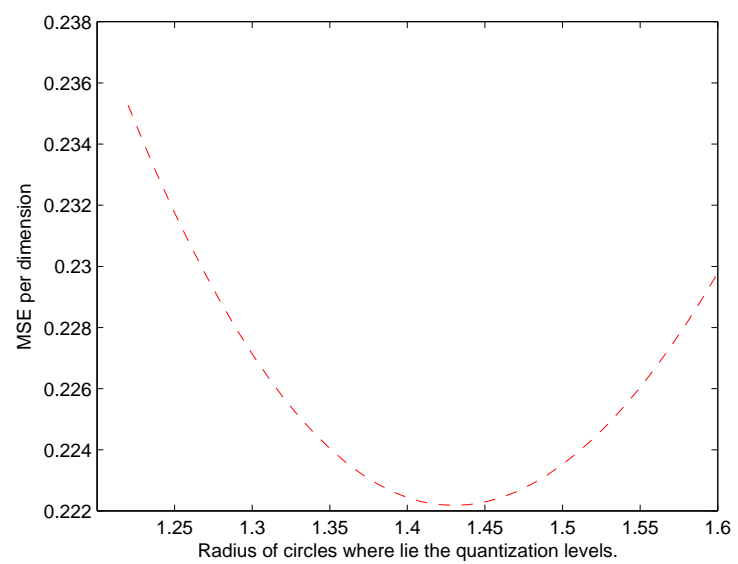

Fig. 15. MSE per dimension for quantization of 10000 samples from uni-variance circular Gaussian distribution.
1) The property of optimal solutions is considered as follows. Assume MMSE per dimension is the objective. For $N=7=1+6$ with two magnitude levels (i.e. the first magnitude quantization level is quantized with one phase quantization level, the second magnitude quantization level with six phase quantization levels), MSE per dimension performance on uni-variance circular Gaussian is shown in Fig. 15 with respect to different radius of circles where lies the second magnitude quantization level. The radius shown in Fig. 15 starts from the second quantization level of Lloyd-Max quantizer for univariate Gaussian around 1.224. Then MSE per dimension decreases with the increase of radius, reaches its unique minimum around 1.43, and then increases with the increase of radius. With more magnitude levels and more than one radiuses needed to be tuned for the optimal performance, there are definitely local minima. But the optimal radiuses could be easily and quickly found with values starting from the quantization levels of Lloyd-Max quantizers for univariate Gaussian.

2) For the quantization lattices with the same number of magnitude quantization levels, the radiuses of the optimal magnitude levels increase with the number of quantization levels $N$, and are saturated with relatively large $N$. The optimal radiuses of the second magnitude quantization level are shown by the vertical coordinates of points in in Fig. 16 corresponding to the number of quantization levels $N=5(=1+4), 6(=1+5), 7(=1+6), 8(=$ $1+7), 9(=1+8)$. They increase with $N$, and gradually slow down. This gives us a guidance on how to tune the optimal magnitude quantization levels.

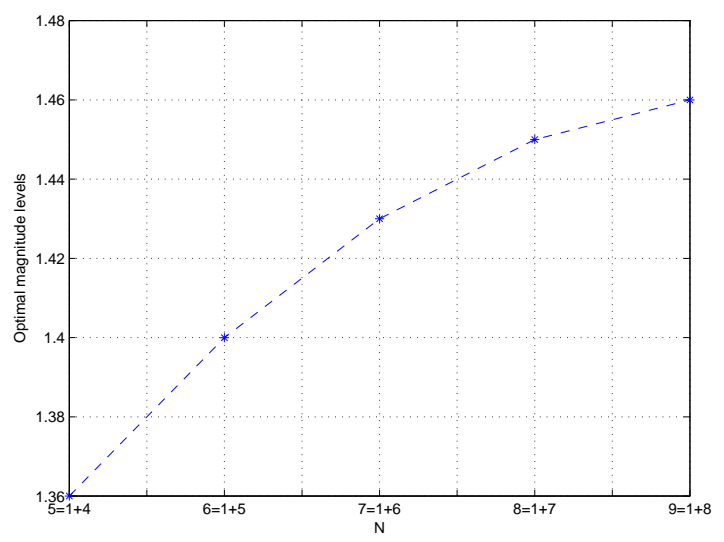

Fig. 16. Optimal magnitude levels for different number of quantization levels for uni-variance circular Gaussian distribution. 


\section{B. Circular Gaussian Distribution}

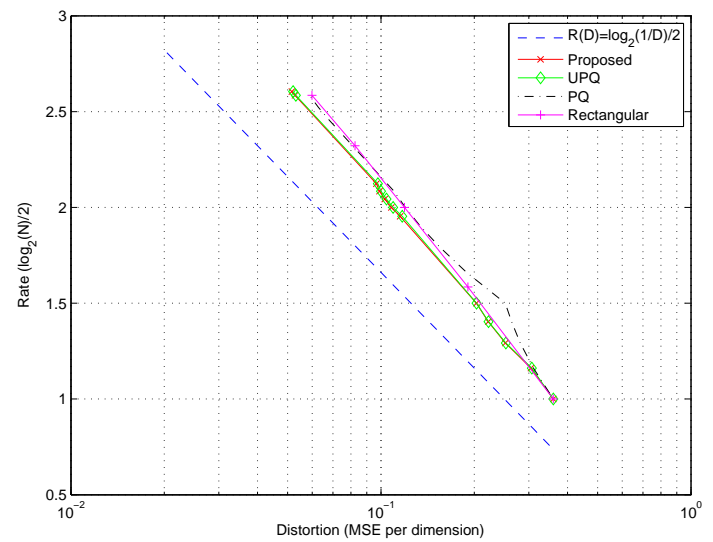

Fig. 17. Rate-Distortion comparison among different quantizers for circular Gaussian distribution.

We show the R-D performance of different quantizers on a uni-variance circular Gaussian distribution in Fig. 17. From Fig. 17, we can see that the R-D performance of our proposed quantizers is always a little better than that of UPQs, and much better than that of PQs and that of Rectangular quantizers. They have the same R-D performance when $N=4$, due to the same quantization level. Rectangular quantizers may have better performance than PQs with some $n^{2}$ quantization levels.

\section{Elliptical Gaussian Distribution}

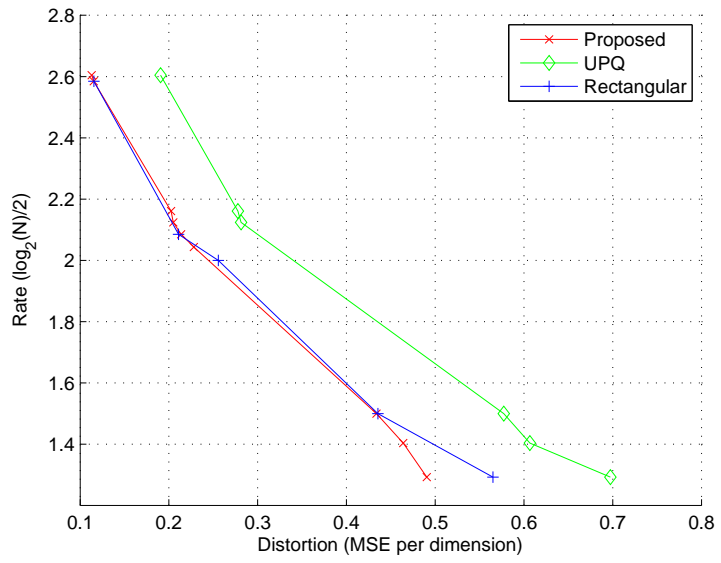

Fig. 18. Rate-Distortion comparison among different quantizers for elliptical Gaussian distribution.

We show the R-D performance of different quantizers on an elliptical Gaussian distribution in Fig. 18. From Fig. 18, we can see that UPQs does not consider the different variances among different random vector components, thus do not perform well. Our proposed quantizers almost always perform better than rectangular quantizers, except when $N=8$. Since $N=8=4 \times 2$ is the best factorization for the rectangular quantizer on elliptical distributions when ratio of data component variances equals 2 . Whereas, for other $N$ non-factorable, rectangular quantizers perform much worse than polar quantizers and the proposed quantizers as expected, although we did not plot it in the figure.

\section{Circular Laplace Distribution}

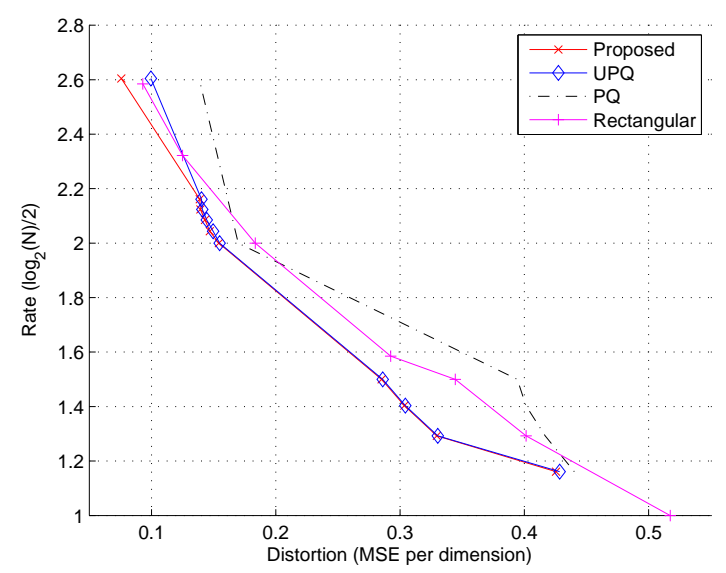

Fig. 19. Rate-Distortion comparison among different quantizers for circular Laplace distribution.

We show the R-D performance of different quantizers on a uni-variance circular Laplace distribution in Fig. 19. It indicates in Fig. 19 that our proposed quantizers always perform a little better than UPQs, and much better than PQs and rectangular quantizers.

\section{E. Elliptical Laplace Distribution}

We show the R-D performance of different quantizers on an elliptical Laplace distribution in Fig. 20. It tells in Fig. 20 that our proposed quantizers always perform better than UPQs, and better than rectangular quantizers except when $N=8=4 \times 2$. Our proposed quantizers have predominant advantages when $N=7,19,37, \cdots$.

\section{F. Bit-rate Saving}

We also evaluate the average bit-rate saving of our quantizers comparing to other quantizers. Average bitrate is calculated by using Bjontegaard's method [20], [21] with fitting polynomials of degree 3 . Bit-rate saving is evaluated based on relative average bit-rate in percentage as shown in the following equation.

$$
\frac{R_{c}-R_{p}}{R_{p}} \times 100 \%
$$




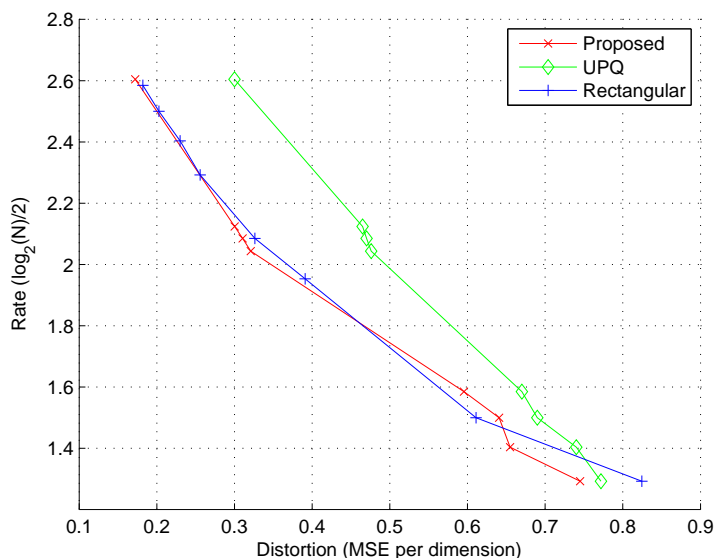

Fig. 20. Rate-Distortion comparison among different quantizers for elliptical Laplace distribution.

TABLE I

AVERAGE BIT-RATE SAVING OF THE PROPOSED QUANTIZERS OVER OTHER QUANTIZERS.

\begin{tabular}{|c|c|c|c|}
\hline & UPQ & PQ & Rectangular \\
\hline Circular Gaussian & $0.36 \%$ & $6.78 \%$ & $3.22 \%$ \\
\hline Elliptical Gaussian & $22.4 \%$ & - & $16.9 \%$ \\
\hline Circular Laplace & $0.94 \%$ & $24.5 \%$ & $5.62 \%$ \\
\hline Elliptical Laplace & $19.8 \%$ & - & $6.32 \%$ \\
\hline
\end{tabular}

where $R_{c}$ is the average bit-rate of the compared quantizers, and $R_{p}$ is the average bit-rate of the proposed quantizers.

From Table I, we can see that the proposed quantizer saves $0.4 \%-24.5 \%$ bit-rate on average, compared to unrestricted polar quantizers, restricted polar quantizers and rectangular qantizers. We did not list the average bit-rate gain over restricted polar quantizers for elliptical distributions, which is even higher than that over unrestricted polar quantizers.

\section{CONCLUSIONS}

In this paper, we proposed a scheme to use transformation plus scalar quantization to replace the optimal vector quantization. The unitary transforms rather than scaling transforms were needed for the optimal vector quantizer approximation. After transformation, scalar quantization for both circular and elliptical distributions was studied in the proposed tri-axis coordinate system. The optimal quantization levels were found in the elastic hexagonal lattices, which included the optimal and the progressive quantizer lattice patterns. The experimental results showed that our proposed quantizers almost always had better performance than UPQs, PQs and rectangular quantizers on both Gaussian and Laplace distributions, especially with prime number of quantization levels. We achieved $O\left(N^{2}\right)$ design complexity and $0.4 \%-24.5 \%$ bitrate saving, where $N$ is the number of quantization levels per dimension. Therefore, we claimed that transforms plus scalar quantizers could approximate the optimal vector quantizers in terms of R-D performance but with much less computational complexity. Our future work will focus on the optimal vector quantizer approximation in high dimensional spaces, and the applications in image and video coding.

\section{REFERENCES}

[1] R. M. Gray and D. L. Neuhoff, "Quantization," IEEE Transactions on Information Theory, vol. 44, no. 6, pp. 2325-2383, 1998.

[2] A. Gersho and R. Gray, Vector quantization and signal compression. Kluwer, 1993.

[3] T. Cover and J. Thomas, Elements of information theory. Wiley, 2006.

[4] W. Bryc, The normal distribution: characterizations with applications. Springer-Verlag, 1995.

[5] T. Cover, J. Thomas, J. Wiley et al., Elements of information theory. Wiley Online Library, 1991, vol. 306.

[6] J. Huang and P. Schultheiss, "Block quantization of correlated Gaussian random variables," IEEE Transactions on Communications Systems, vol. 11, no. 3, pp. 289-296, 1963.

[7] J. Max, "Quantizing for minimum distortion," IRE Transactions on Information Theory, vol. 6, no. 1, pp. 7-12, 1960.

[8] P. Zador and S. U. D. of Statistics, Development and evaluation of procedures for quantizing multivariate distributions. Dept. of Statistics, Stanford University, 1963.

[9] A. Gersho, "Asymptotically optimal block quantization," IEEE Transactions on Information Theory, vol. 25, no. 4, pp. 373-380, 1979.

[10] P. Moo and D. Neuho, "Uniform polar quantization," in in Proceedings of IEEE International Symposium of Information Theory. Citeseer, 1998.

[11] W. Pearlman, "Polar quantization of a complex Gaussian random variable," IEEE Transactions on Communications, vol. 27, no. 6, pp. 892-899, 1979.

[12] J. Bucklew and N. Gallagher Jr, "Quantization schemes for bivariate Gaussian random variables," IEEE Transactions on Information Theory, vol. 25, no. 5, pp. 537-543, 1979.

[13] - "Two-dimensional quantization of bivariate circularly symmetric densities," IEEE Transactions on Information Theory, vol. 25 , no. 6, pp. 667-671, 1979.

[14] S. Wilson, "Magnitude/phase quantization of independent Gaussian variates," IEEE Transactions on Communications, vol. 28, no. 11, pp. 1924-1929, 1980.

[15] P. Swaszek and J. Thomas, "Optimal circularly symmetric quantizers," Journal of the Franklin Institute, vol. 313, no. 6, pp. 373-384, 1982.

[16] Q. Chen and T. Fischer, "Robust quantization for image coding and noisy digital transmission," in Proceedings on Data Compression Conference, 1996. IEEE, 1996, pp. 3-12.

[17] A. Jain, Fundamentals of digital image processing. PrenticeHall, Inc. Upper Saddle River, NJ, USA, 1989.

[18] E. Ravelli and L. Daudet, "Embedded polar quantization," Signal Processing Letters, IEEE, vol. 14, no. 10, pp. 657-660, 2007.

[19] E. Barnes and N. Sloane, "Optimal lattice quantizer in three dimensions." SIAM Journal of algebraic discrete math., vol. 4, no. 1 , pp. $30-41,1983$.

[20] G. Bjontegaard, "Calculation of average psnr differences between rd-curves (vceg-m33)," in in Proceedings of VCEG Meeting (ITU-T SG16 Q. 6), 2001, pp. 2-4.

[21] S. Pateux and J. Jung, "An excel add-in for computing bjontegaard metric and its evolution," in in Proceedings of VCEG document VCEG-AE07, 31st VCEG Meeting, Marrakech, MA, 2007, pp. 15-16. 


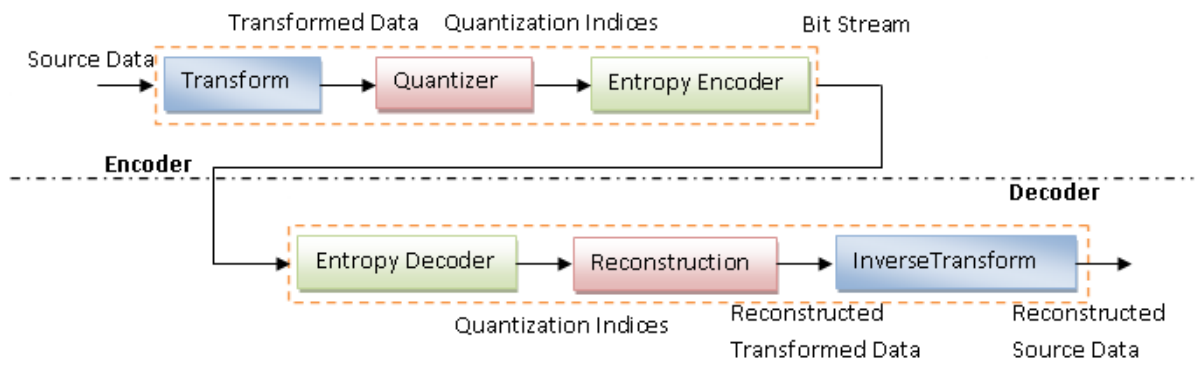

Fig. 1. General encoding and decoding pipeline with transforms and scalar quantization.

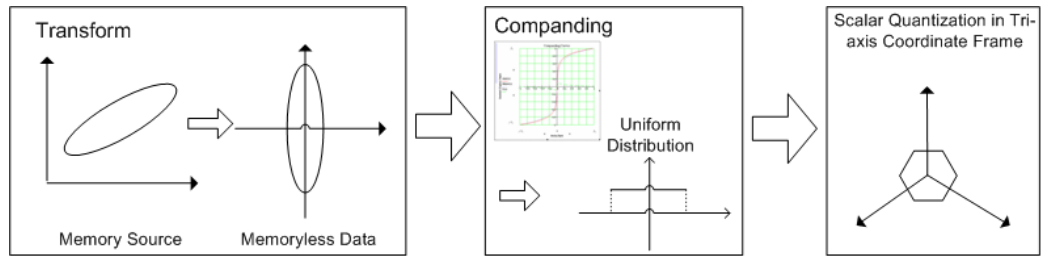

Fig. 3. Transform plus scalar quantization with companding technique Pipeline.

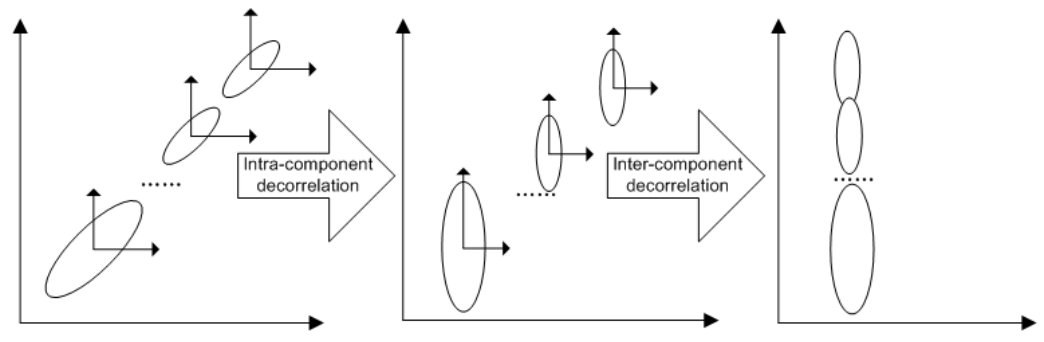

Fig. 4. Gaussian mixture model decorrelation. 